\title{
Planning for the pandemic
}

\author{
Joanne Embree MD FRCPC
}

$\mathrm{O}$ ver the past year, many of us have become involved in the development of strategic plans for the hospitals, health authorities and clinical practices in which we work in case an influenza pandemic occurs in the very near future.

Planning for this on the front lines is difficult, due in large part to the uncertainties involved. How will the pandemic evolve? Is the current H5N1 strain of bird flu going to be 'the' one? How closely will the pandemic resemble that of 1918 ? Will it have the same transmission characteristics as the yearly endemic influenza strains or will it be so different that our routine infection prevention precautions for influenza will be ineffective? Will there be a useful vaccine that is widely available and safe? That the current death rate associated with H5N1 strain infections in humans is approximately $60 \%$ is quite frightening; an influenza pandemic with such a high death rate is almost incomprehensible. Therefore, it is a relief to hear that the upper estimates are at a much lower rate of approximately $5 \%$ in most suggested epidemiological models. Will the use of oseltamivir really work to prevent infection, illness, morbidity or death? If so, will there be sufficient supplies available in Canada? How are we supposed to make plans so that our medical system, which is already quite stressed, will be functional under the extreme conditions that are anticipated? One major difficulty is that we do not actually know how soon, if at all, the pandemic will occur. Specific, highly detailed plans made today may not be applicable in the future. As a result, most contingency plans are being made for a generic situation based on the general assumption that some percentage of the workforce will be absent from work for some specified period of time. In general, the plans tend to be impersonal because they concentrate on essential functions that need to be undertaken in an institution and assume that, with training, all personnel can cross-cover these services to accommodate for those times when the employees who routinely perform those tasks are absent.

With our already very busy professional lives, many are not sure how much time and effort we really should devote to contingency planning for something that may not happen for a few years, if at all. Having said that, we all know that it is better to be well prepared. We really do need to take the time and make the needed effort to plan this correctly. In general, systems that do not work well before a crisis are unlikely to function well when a crisis develops. No two medical institutions are identical; therefore, each institution will have to develop contingency plans that are specific to the unique administrative structure and needs of that institution. This is a complex undertaking with a large number of factors to take into consideration, and many institutions are in the process of restructuring administrative systems or remodelling physical structures. On the positive side, it is possible that we may actually be able to solve a few existing problems that routinely plague us during this process. Therefore, now would be the time to encourage those in charge to critically look at the inefficiencies or deficiencies in our health care systems that will affect how well we care for patients with infections, and take steps to correct them. If the infection prevention and control procedures that will be necessary during an influenza epidemic are similar to those that are routinely and appropriately used, then ensuring their use during the pandemic will be much easier.

One issue related to the response to an influenza pandemic that has received some attention recently is the question of how health care professionals will balance their professional and personal responsibilities during the epidemic. There is the fear that a significant number will not report to work or will insist on working only in 'safe' situations. Hurricane Katrina taught us all that the pressure to care for one's family or one's pet is very significant during crisis situations. Severe acute respiratory syndrome taught us that tired, overworked health care workers are at an increased risk of acquiring the infections from their patients. Knowing this, and assuming an influenza pandemic, the potential to then transmit an infection to one's family is substantial. These factors will clearly need to be considered as we help institutions plan for the pandemic. During that process, it will be advisable to also reflect on how each of us may personally react to what will likely be a very stressful situation. We do not know which of our colleagues, friends and family members will be affected. It is hard to comprehend that some of those who we know and love may actually die. Clearly, we all have a moral and professional obligation to ensure that our patients are cared for appropriately. This is a responsibility that should be shared among all of the health care providers in a group or institution. However, it will be important to realistically anticipate the specific personal and family needs of our colleagues and include ways to help meet them.

In all likelihood, there will be an influenza pandemic in the near future. With appropriate planning, we can hopefully prevent it from being as devastating as some have predicted. 


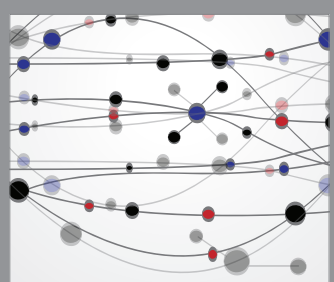

The Scientific World Journal
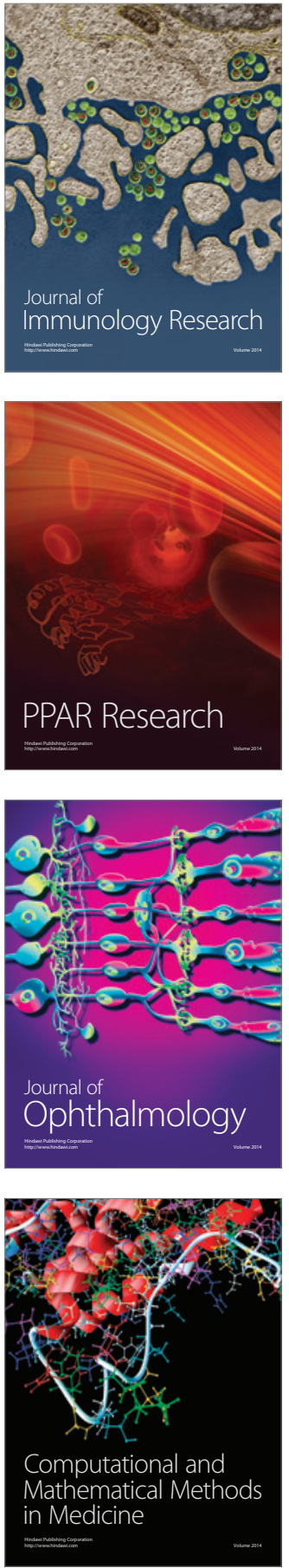

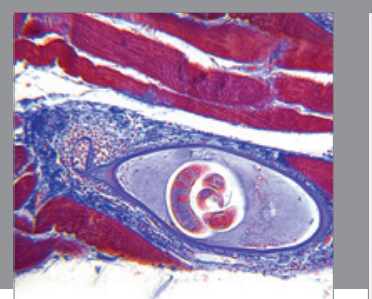

Gastroenterology Research and Practice

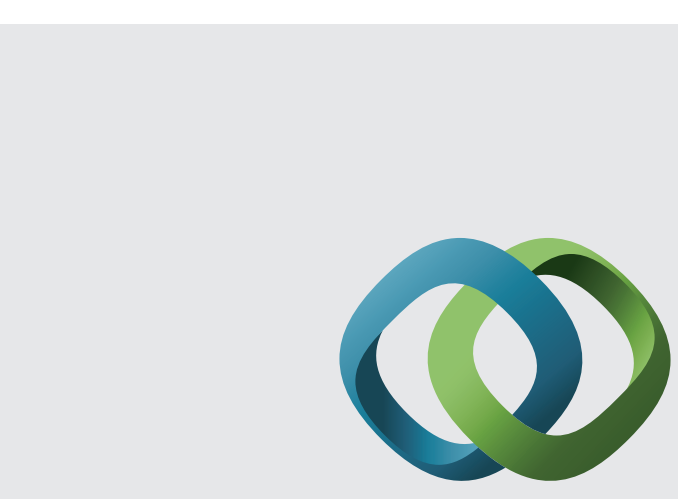

\section{Hindawi}

Submit your manuscripts at

http://www.hindawi.com
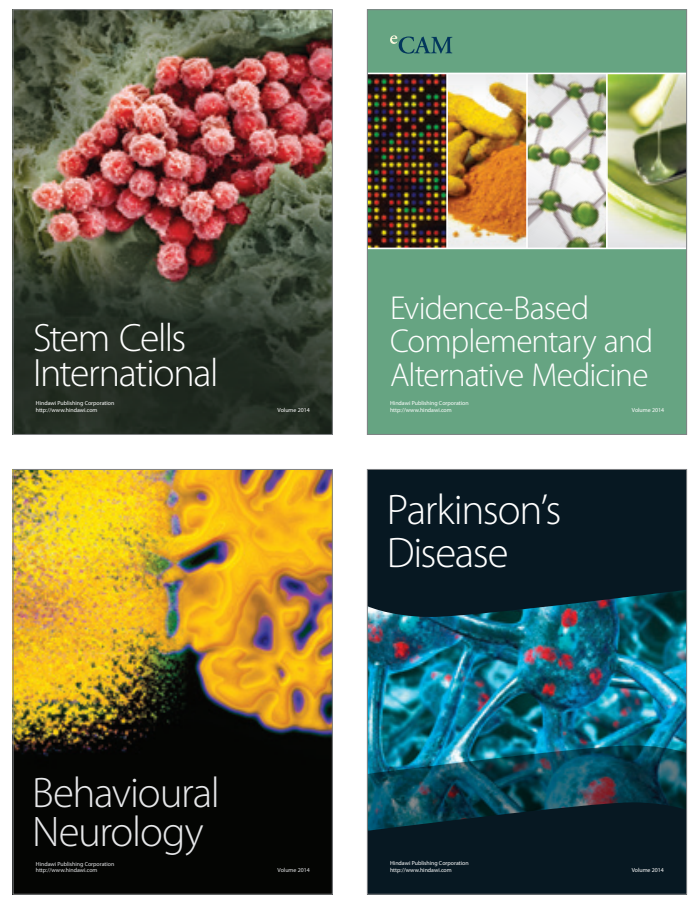
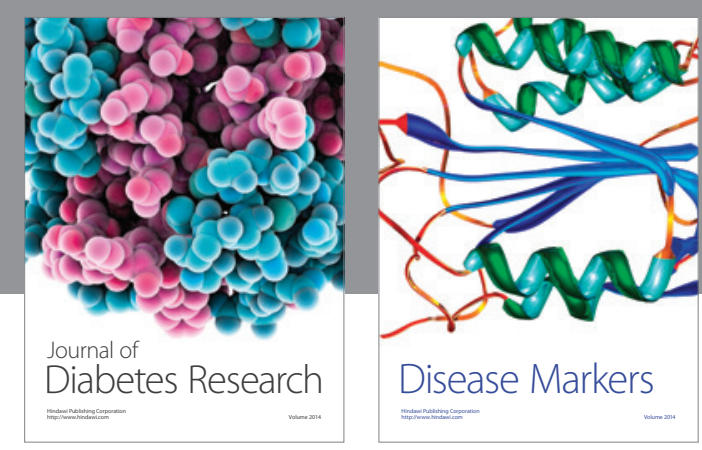

Disease Markers
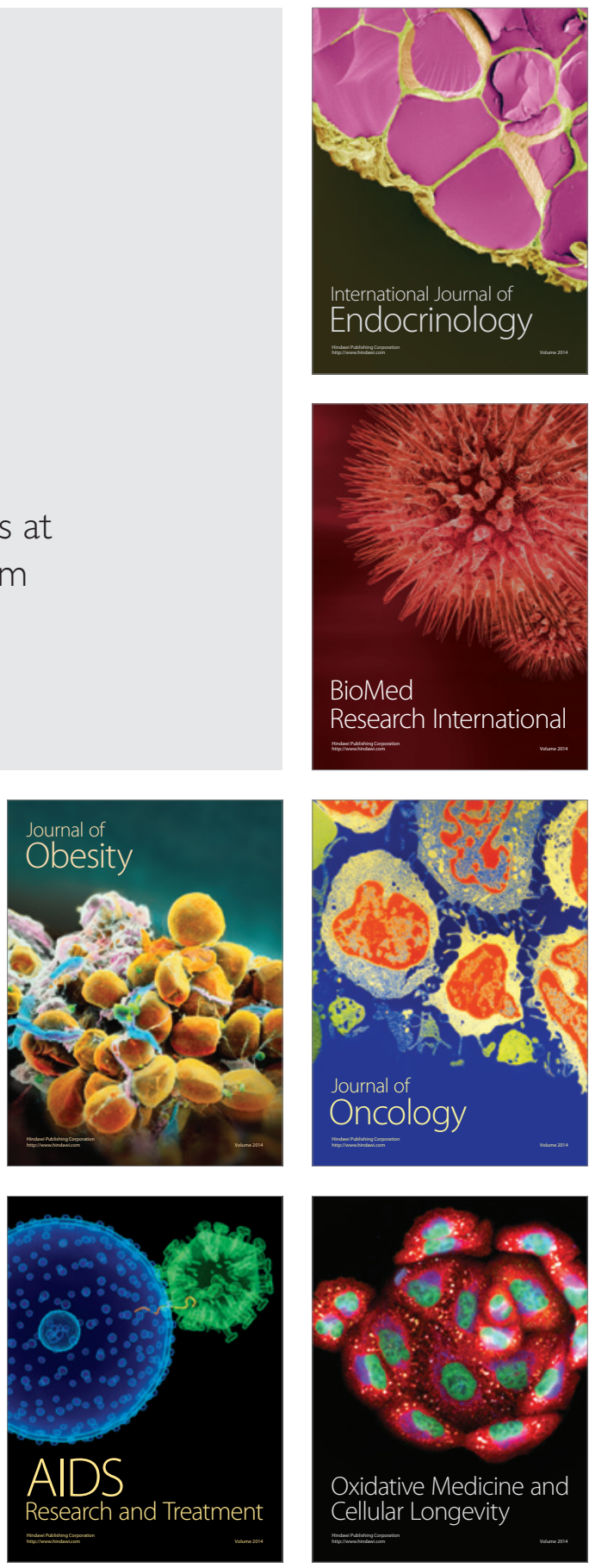